\title{
Intervening in the Acute Phase of Postradiation Carotid Blowout Syndrome
}

\author{
Marrigje A. de Jong ${ }^{1 \oplus}$ Carlos Candanedo ${ }^{2}$ Menachem Gross ${ }^{1}$ José E. Cohen ${ }^{2,3}$ \\ ${ }^{1}$ Department of Otolaryngology/Head and Neck Surgery, Hadassah- \\ Hebrew University Medical Center, Jerusalem, Israel \\ 2 Department of Neurosurgery, Hadassah-Hebrew University Medical \\ Center, Jerusalem, Israel \\ 3 Division of Endovascular Neurosurgery and Interventional \\ Address for correspondence Dr. Marrigje A. de Jong, MD, Department \\ of Otolaryngology/Head and Neck Surgery, Hadassah Hebrew- \\ University Medical Center, Jerusalem, 91120, Israel \\ (e-mail: dejong.marije@gmail.com).
} Neuroradiology, Hadassah-Hebrew University Medical Center,

Jerusalem, Israel

Int Arch Otorhinolaryngol 2019;23:172-177.

\begin{abstract}
Introduction Acute carotid blowout syndrome (aCBS) is a severe complication of head and neck cancer (HNC). It can be defined as a rupture of the extracranial carotid arteries, or one of their branches, that causes life-threatening hemorrhage, and which nowadays can be treated with urgent endovascular intervention.

Objective We retrospectively evaluate the endovascular management of aCBS and its outcome in years of survival.

Methods Retrospectively, we describe our experience with endovascular control of aCBS in patients treated for HNC. We review the characteristics, pathology, endovascular treatment and morbidity and assess the gain in life years.

Results Nine individuals were included in this study. Four patients had been previously diagnosed with laryngeal squamous cell carcinoma (SCC), one with paranasal SCC, one with nasopharyngeal carcinoma and three with oral or maxillary adenocarcinoma. All subjects underwent radiotherapy and surgical excision to different extents. Twelve endovascular procedures were performed for injuries to the internal carotid artery $(n=3 ; 25 \%)$, external

Keywords

- carotid arteries

- endovascular procedures

- radiotherapy

- head and neck neoplasms carotid artery $(n=1 ; 7 \%)$ or one of their branches $(n=8 ; 67 \%)$. Deconstructive methods were used in nine procedures, and three procedures were mainly reconstructive with deployment of covered stents. Total control of bleeding was achieved in all individuals with no intraprocedural complications.

Conclusion Endovascular therapy is an effective alternative for the management of exsanguinating CBS. In our series, this palliative therapy increased the overall patient survival by an estimated 9 months.
\end{abstract}

\section{Introduction}

Carotid blowout syndrome (CBS), although rare, is one of the most severe complications of head and neck cancer (HNC). It can be defined as a rupture of the extracranial carotid arteries or one of their branches and occurs in 6 to $10 \%$ of

(1) Marrigje A. de Jong's ORCID is https://orcid.org/0000-00030654-3711.

received

January 24, 2018

accepted

November 13, 2018

published online

March 1, 2019 ISSN 1809-9777. advanced HNC cases. ${ }^{1,2}$ Patients who received radiation therapy, who developed postoperative wound complications or fistulation, and those with tumor recurrence are at higher risk. We know that radiation, in special, affects the relative superficial vascular structures of the head and neck. A high radiation dose needs to be given to achieve local control and prevent recurrence; however, this damages the arterial wall, which eventually cannot sustain its integrity against pressure.

Copyright @ 2019 by Thieme Revinter

Publicações Ltda, Rio de Janeiro, Brazil

License terms

$10.1055 / \mathrm{s}-0038-1676660$. 
Carotid blowout is a syndrome that can be classified in three subtypes according to its clinical severity; 1 ) threated CBS, in which the carotid artery is exposed to the outer environment and physical or radiologic examination are suggestive for inevitable carotid hemorrhage in the immediate future if no action is taken 2) impending CBS presents as sentinel hemorrhages that resolve spontaneously or with packing and 3) acute CBS (aCBS), in which a non-self-limiting profuse bleeding develops. Acute carotid blowout syndrome is life threatening and needs urgent intervention. Historically, it was treated by head and neck surgeons in the operating room by ligation or repair of the carotid artery. However, to identify the source of bleeding in such cases is difficult because of tumor bulk, postradiation fibrosis or coexisting infection, and, therefore, surgical control of bleeding is often technically not possible. Secondly, this treatment has a mortality as high as $40 \%$ and morbidity of $60 \%$, as mentioned in the literature ${ }^{2}$. In recent years, there has been a shift to endovascular intervention as first line of treatment for aCBS in many medical centers. Improved techniques with liquid adhesives, coils and stents might result in more favorable direct outcomes today.

We thought it worthwhile to review the endovascular diagnostic and therapeutic interventions of patients with postradiation aCBS and a medical history of HNC who presented in the last few years to the Endovascular Neurosurgery Department of Hadassah-Hebrew University Medical Center. We also evaluated the direct and long-term neurological outcomes in those patients.

\section{Methods}

We conducted a search of our patient files over the past 10 years. We selected patients with a medical history of HNC who received surgical treatment and chemoradiation or radiation therapy alone and presented to our medical center with a profound and active transcervical or transoral bleeding. Patients with traumatic aCBS were excluded. All patients were initially seen by the otolaryngology physician on call and when aCBS was suspected, they were urgently transferred to the endovascular neurosurgery unit. They were evaluated by means of a complete neuroangiogram of the supra-aortic arteries and their branches. The acute scenario in these cases did not allow for performing a balloon occlusion test to assess the risk of ischemic cerebrovascular accident (CVA); however, the patients were evaluated for complete or incomplete circle of Willis. The site of bleeding was defined either as internal carotid artery (ICA), external carotid artery (ECA) or one of their major branches. The severity of the vascular lesion was classified as grade 1 to 4 , in which grade 1 meant increased vascular brushes, grade 2 focal vascular irregularities, grade 3 a pseudoaneurysm and grade 4 vascular extravasation (-Fig. 1). Depending on the site and severity of the lesion, they received urgent endovascular treatment to control the bleeding by either a deconstructive or reconstructive method.

Angiography: under local or general anesthesia, a transfemoral arterial approach was used, and a French catheter with size between 4 and 9 french was placed on the right femoral artery with ultrasound guidance. Selective angiograms were obtained from the common, internal and external carotid arteries and their branches. Dynamic images were evaluated for active hemorrhage with extravasation of contrast, pseudoaneurysm, vascular irregularities or brushes, A$\mathrm{V}$ fistula or stenotic vasculopathy.

Deconstructive methods result in complete occlusion of the carotid artery segment. Two micro-catheters were inserted, one distal and one proximal to the lesion. After inflation of a detachable balloon proximal to the lesion, either detachable coils or micron embospheres were deployed. In some cases, a liquid embolic material, Onyx (EY 3 Inc. Meditronics), was injected through the distal catheter, either directly or in the interstices of coils while the balloon was pulled out gently. At the end of the procedure, digital subtraction angiography was performed to evaluate vascular deconstruction. Successful deconstructive management was achieved when complete obliteration of the lesion with clinical hemostasis was noted.

Reconstructive methods avoid the risk of major cerebral infarction by maintaining the blood flow by deployment of a stent across the ruptured segment. After a guiding sheath was placed at the right segment, a covered stent was introduced and appropriately deployed ( - Fig. 2D). Evaluation for leak was performed immediately and 15 minutes after deployment of the stent. Adequate coverage of the lesion and clinical hemostasis indicated successful revascularization management. At the conclusion of reconstructive vascular treatment, patients received anticoagulation therapy accordingly.

Subsequently, the characteristics of each individual, the tumor location, tumor pathology, local recurrence and tissue factors as well as the origin of the bleeding were assessed for each individual separately. The methods and outcomes of the endovascular interventions were reviewed, and, as a final step, we assessed the gain in life years. No statistical comparison was done considering the small number of cases.

\section{Results}

A total of 9 individuals were included in our series, 1 female and 8 male subjects with a mean age of $60 \pm 12$ standard deviation (SD) years-old. Their characteristics and pathology are shown in -Table 1. Five patients had a previous medical history positive for squamous cell carcinoma (SCC), located in the larynx $(n=4)$ or in paranasal tissues $(n=1)$. One patient had been previously diagnosed with nasopharyngeal carcinoma and three patients had oral maxillary adenocarcinoma. All subjects received radiation therapy, and, except for one patient, they all underwent surgical excision to different extents. Despite this treatment, 7 patients presented with recurrence within 1 to 13 years (median 3 years; mean 4.6 years) after diagnosis. In total, there were 12 endovascular procedures performed in our series in the period between May 2012 and January 2015. End point of follow-up for those still alive was March 2015. The time interval between initial diagnosis and the event of aCBS varied from 2 to 30 years, and we noticed that patients with laryngeal SCC had a shorter interval (mean 4.25 years) compared with the patients with 

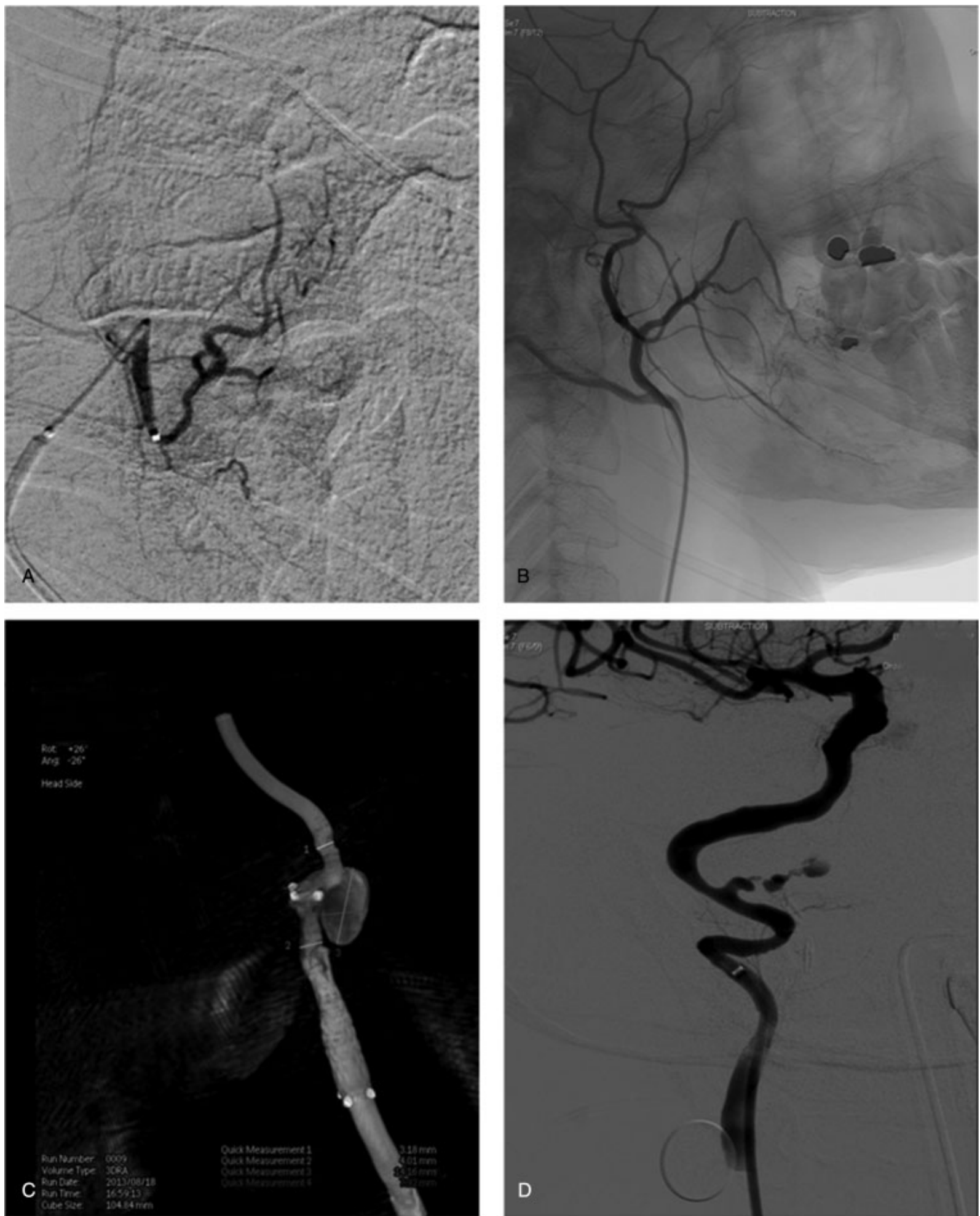

Fig. 1 Angiography severity. (A) grade 1 vascular brushes (B) grade 2 vascular irregularity of the right palatine artery. (patient M45) (C) grade 3 pseudoaneurysm of the left internal carotid artery (patient M57) (D) grade 4 vascular extravasation of the petrous segment of the right internal carotid artery (patient F45).

oral and maxillary adenocarcinoma or nasopharyngeal carcinoma (mean 14 years), even though there was no statistically significant difference $(p=0.10)$. Eight patients presented with aCBS to the emergency department. Upon arrival, they were evaluated according to the ABC-method (airway, breathing, cardiac) and treated accordingly. One patient underwent a CT- angiography prior to endovascular angiography; all others were directly transferred to the endovascular neurosurgery unit. One patient developed an acute and uncontrolled bleeding during admission to the oncology department. The patients presented with injuries on the ICA $(n=3 ; 25 \%)$, ECA $(n=1 ; 7 \%)$, or their branches $(n=8 ; 67 \%)$, that were 

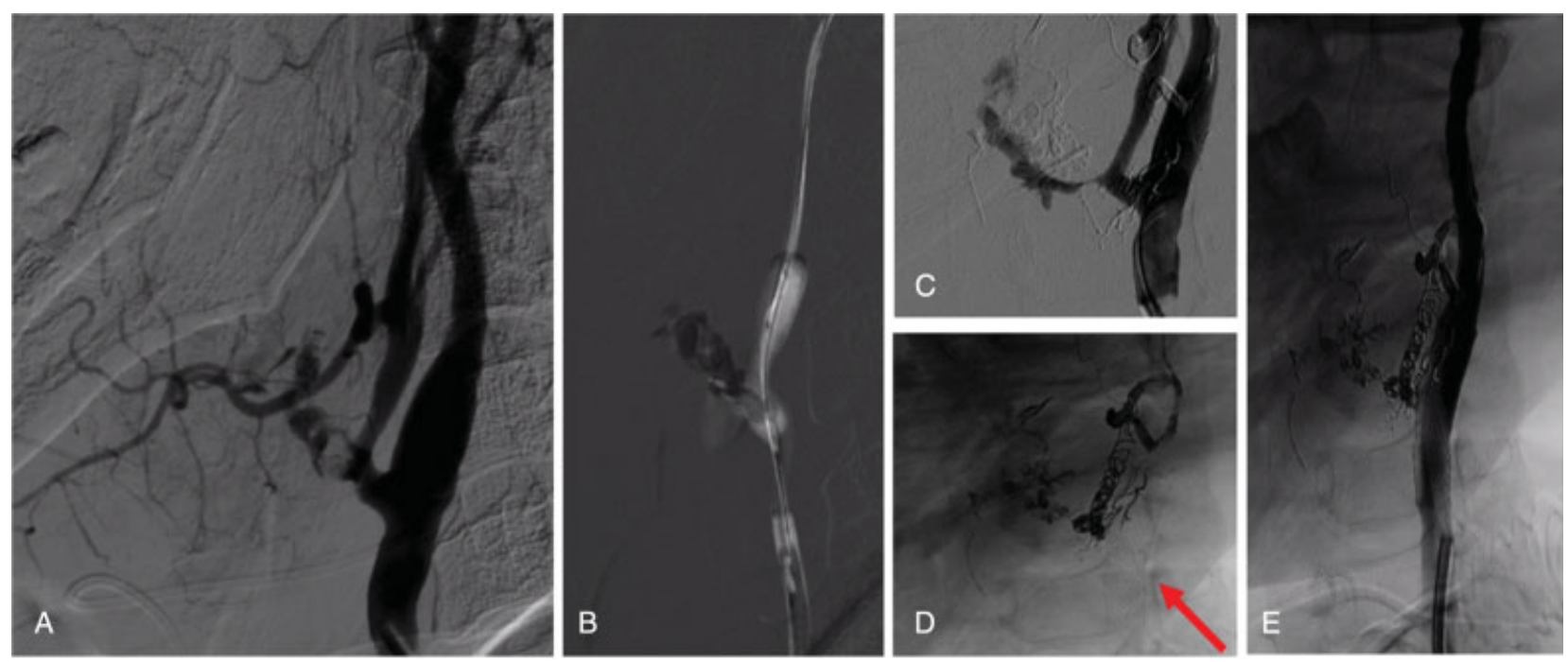

Fig. 2 Stenting for grade 4 acute carotid blowout syndrome of the left external carotid artery (patient M50) A 50-year-old male who developed acute carotid blowout syndrome grade 4 with extravasation of the left external carotid artery 5 years after total laryngectomy and radiation therapy (A). Liquid embolic material was injected in the left ECA (B) a remaining leak was noticed during reconstruction (C) coils were placed in the left external carotid artery and a covered stent was introduced in the left common carotid artery/internal carotid artery near the injured segment (D). A reconstruction angiogram showed control of bleeding with intact blood flow through the internal carotid artery (E).

described as vascular brushes $(n=5)$, focal vascular irregularity $(n=1)$, pseudoaneurysm $(n=3)$ and/or active extravasation $(n=3)$. Two subjects required bilateral treatment. Deconstructive methods were used in nine procedures; one procedure was fully reconstructive and in two other procedures both methods were used. Regarding the treatment materials, in three procedures liquid embolic Onyx (EY 3 Inc. Meditronics) was required for embolization while micron embospheres were used in five cases. Detachable coils were used in seven procedures and three procedures involved covered stents. There were four complex cases in which subjects were treated with two or three different materials (-Fig. 2). Total control of the bleeding was achieved in all individuals $(100 \%)$ with no intraprocedural complications (0\%). Three patients required twice angio-embolization because of recurrent CBS, respectively within 2, 11 and 98 days. However, rebleeding occurred at a different site. In our study population, the evaluation of stent patency was complicated by the morbidity and mortality of disease and was, therefore, often not performed. Seven patients died within a 2-year period. The estimated average survival was 9 months in our population. Two patients are still alive after 27 and 7 months, respectively, from the procedure.

\section{Discussion}

Acute carotid blowout syndrome is a catastrophic event with life-threatening hemorrhage of the extracranial carotid artery and its major branches, and, although rare, effort should be made to prevent such a dangerous condition. Several studies have tried to determine predisposing factors for development, morbidity and mortality of aCBS, such as tumor site, staging, pathologic type, local recurrence, radiotherapy, postoperative inflammation and fistulation, poor nutrition, origin of bleed- ing, timing and type of intervention. ${ }^{2-5}$ Small study samples and a broad diversity in pathology and treatment, as in this review of cases, make it difficult to find significant outcomes.

Recurrence of HNC is as high as 30 to $50 \% .^{6} \mathrm{~A}$ second primary tumor occurs in $20 \%$ of cases. In our series 7 out of 9 (78\%) patients presented with recurrence of malignant disease. Re-irradiation is one of the treatment options, although this results in a radiation dose that can exceed the normal tissue tolerance. A radiation dose of $>66$ Gray (Gy) to the head and neck region results in better local control but increases the risk for mucosal damage, cranial nerve injury, tissue necrosis, inflammation including radiation arteritis and carotid stenosis. ${ }^{7}$ More specifically, radiation causes obliteration of the vasa vasorum, adventitial fibrosis, premature atherosclerosis and weakening of the arterial wall. ${ }^{8} \mathrm{~A}$ damaged arterial wall cannot sustain its integrity against pressure and, eventually, bleeding can occur. ${ }^{4}$ The latent period to CBS can be as short as several weeks and as long as several years. ${ }^{3,7}$ It depends not only on the total radiation dose, but also on local tissue factors and usually occurs in premature atherosclerotic vessel segments. ${ }^{7}$

To our knowledge, there are no clear guidelines for endovascular management in aCBS. However, we postulate two basic principles: A) acute hemorrhage with extravasation (grade 4) can be controlled best by stenting instead of devascularization. B) injury to the ICA is preferred to be managed with revascularization stenting instead of devascularization. Endovascular stenting is a useful alternative for several reasons, one of them being that it allows for rapid control of the hemorrhage while maintaining cerebral blood flow. However, preservation of a weakened carotid artery is extremely difficult, and a deconstructive method might be easier for external carotid segments or for ICA segments if the contra- or ipsilateral blood flow is intact or may already be compensating. ${ }^{7}$ 


\begin{tabular}{|c|c|c|c|c|c|c|c|c|c|c|}
\hline 范 & $z$ & $z$ & $z$ & $z$ & $z$ & $z$ & $z$ & $z$ & $>$ & 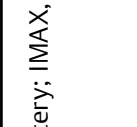 \\
\hline 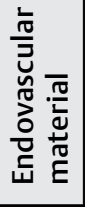 & 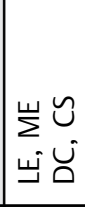 & $\begin{array}{l}u \\
u \\
0 \\
u \\
\exists\end{array}$ & O & 㞫 & $\vec{\Sigma}$ & Ø & 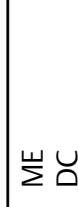 & 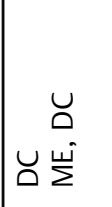 & 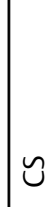 & 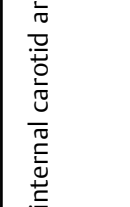 \\
\hline 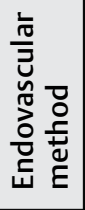 & 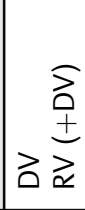 & 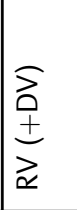 & b & ठे & aे & a & ठ己 & るる & $\gtrsim$ & 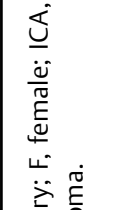 \\
\hline 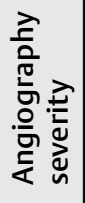 & $-\nabla$ & t & $m$ & - & - & - & $\sim m$ & $m-$ & $\nabla$ & 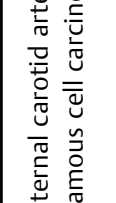 \\
\hline 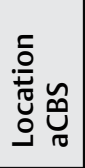 & 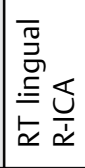 & 壱 & $\frac{\mathbb{U}}{\dot{U}}$ & 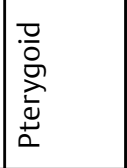 & $\sum_{i}^{\times}$ & 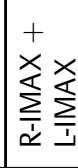 & 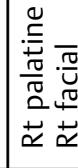 & 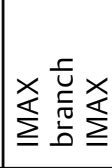 & $\begin{array}{l}\frac{\widetilde{U}}{\alpha} \\
\frac{\alpha}{a}\end{array}$ & 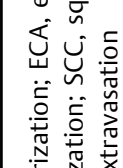 \\
\hline 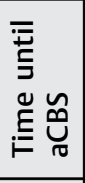 & 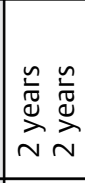 & 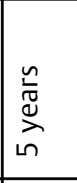 & 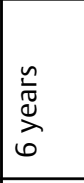 & 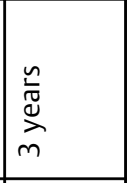 & 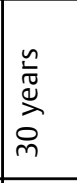 & 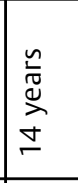 & 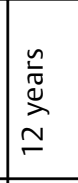 & 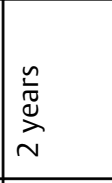 & 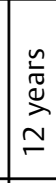 & 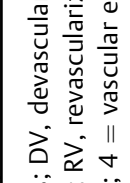 \\
\hline 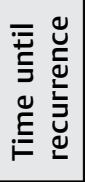 & 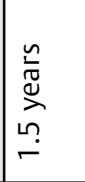 & 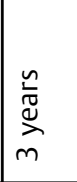 & 1 & 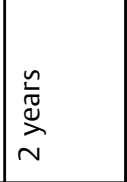 & 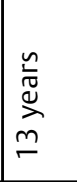 & 1 & 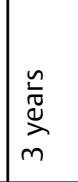 & 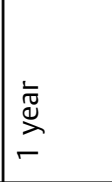 & 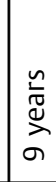 & 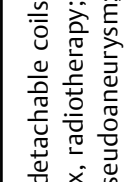 \\
\hline 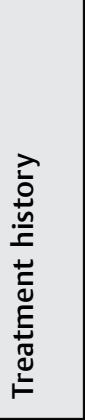 & 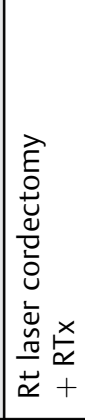 & 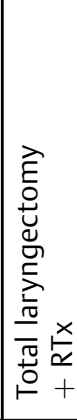 & 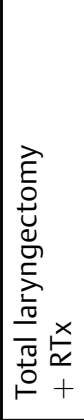 & 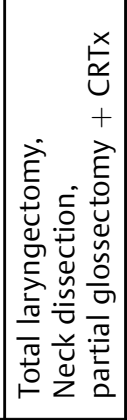 & 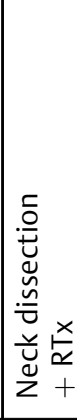 & \begin{tabular}{|l}
$\stackrel{x}{a}$ \\
$\stackrel{a}{a}$
\end{tabular} & 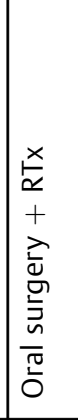 & 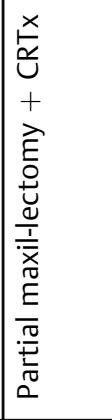 & 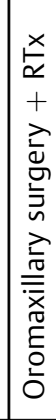 & 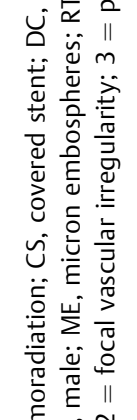 \\
\hline 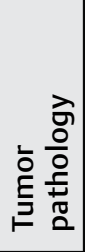 & $\ddot{\sim}$ & $\ddot{\sim}$ & $u$ & $\ddot{\sim}$ & $\ddot{\sim}$ & 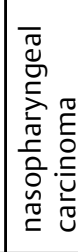 & 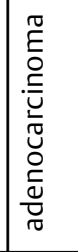 & 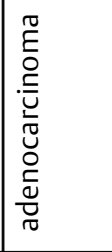 & 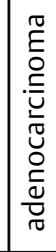 & 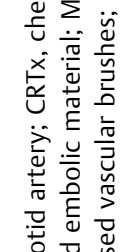 \\
\hline 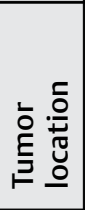 & 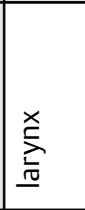 & 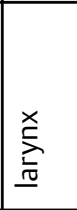 & 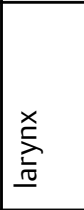 & 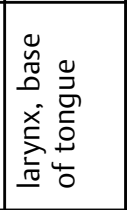 & 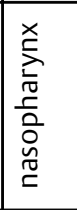 & 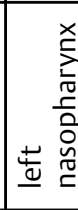 & 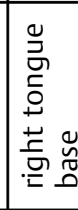 & 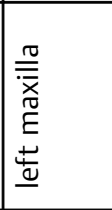 & 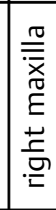 & $\overline{\mathrm{g}}$ \\
\hline 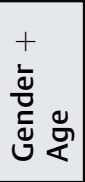 & $\bar{\gamma}$ & ㅇํ & 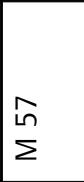 & $\stackrel{N}{\Sigma}$ & $\begin{array}{l}8 \\
\Sigma\end{array}$ & 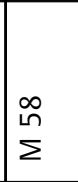 & $\stackrel{\stackrel{\wp}{\sim}}{\Sigma}$ & $\begin{array}{l}\hat{\theta} \\
\Sigma\end{array}$ & $\begin{array}{l}\stackrel{\Perp}{R} \\
\leftarrow\end{array}$ & : \\
\hline
\end{tabular}


Once acute hemorrhage occurs with transcervical or transoral bleeding, there is, in our opinion, no indication for evaluation of the contra- and ipsilateral blood flow by means of a balloon occlusion test, despite the fact that between 15 and $20 \%$ of patients develop immediate or delayed cerebral ischemia after permanent occlusion of the ICA, as mentioned in the literature. ${ }^{9,10}$ A possible explanation for this complication is an incomplete circle of Willis. Therefore, this vascular site initially needs to be evaluated during angiography. In case of doubt, again, we favor the use of covered stents, if possible, to minimize the risk of ischemic stroke.

Some studies report unfavorable long-term outcomes after deployment of a foreign body, such as a covered stent, in a field of ongoing contamination and neoplastic disease. ${ }^{11,12}$ Complications of this technique include local infection, stent occlusion and rebleeding. In our study, we used covered stents in cases of vascular extravasation (grade 4 angiographic severity) for the purpose of rapid bleeding control. Twice the hemorrhage involved the ICA, one case involved the ECA. Both patients who underwent stenting of the ICA died within 1 year after the procedure, and, in one case, there was severe morbidity from a major stroke, despite anticoagulant therapy after the procedure. In the third case, the patient is still alive after 7 months follow-up without complication. Endovascular stent graft reconstruction is good for achieving immediate and temporary hemostasis. Considering the limited long-term survival in patients with $\mathrm{HNC}$, this procedure can be considered palliative.

Rebleeding did occur in three of our cases. These were initially treated with micron embospheres and detachable coils for aCBS from the palatine, lingual and internal maxillary arteries respectively; rebleeding occurred at another vascular site. External carotid branches are not suitable for deployment of a covered stent. The patients had recurrence of malignant disease and they died within 1 to 2 years after aCBS first occurred.

\section{Conclusion}

Head and neck cancer patients with postradiation aCBS are a small and neglected population within today's medicine. However, the severity of this devastating complication drew our attention, especially in view of the recent advantages found with the use of endovascular treatment. Not many studies describe stenting and embolization in these specific cases of aCBS, and, therefore, we ought it worthwhile to describe our experiences. We are aware of the limited number of cases of aCBS in this study and the diversity in the pathology and treatment history of our patients, and, therefore, we cannot offer practical recommendations for treatment. However, we emphasize here that, in experienced hands, embolization and stenting are both safe and successful interventions in the acute phase of CBS. Direct control of bleeding was achieved in all cases. The estimated average survival after endovascular treatment in our series is 9 months. Considering the limited long-term survival in this subset of patients with HNC, these procedures should be considered palliative.

\section{Contributions}

All authors contributed to conception, design, and manuscript preparation as well as reading and approval of the final manuscript.

\section{Conflicts of Interest}

Dr. de Jong, Dr. Candanedo, Dr. Gross and Prof. Cohen report no financial interests or potential conflicts of interest.

\section{Acknowledgments}

The first and second author equally contributed to this study.

\section{References}

1 Roh JL, Suh DC, Kim MR, et al. Endovascular management of carotid blowout syndrome in patients with head and neck cancers. Oral Oncol 2008;44(09):844-850

2 Lu HJ, Chen KW, Chen MH, et al. Predisposing factors, management, and prognostic evaluation of acute carotid blowout syndrome. J Vasc Surg 2013;58(05):1226-1235

3 McDonald MW, Moore MG, Johnstone PA. Risk of carotid blowout after reirradiation of the head and neck: a systematic review. Int J Radiat Oncol Biol Phys 2012;82(03):1083-1089

4 Chen YJ, Wang CP, Wang CC, Jiang RS, Lin JC, Liu SA. Carotid blowout in patients with head and neck cancer: associated factors and treatment outcomes. Head Neck 2015;37(02):265-272

5 Brinjikji W, Cloft HJ. Outcomes of endovascular occlusion and stenting in the treatment of carotid blowout. Interv Neuroradiol 2015;21(04):543-547

6 Hoebers F, Heemsbergen W, Moor S, et al. Reirradiation for headand-neck cancer: delicate balance between effectiveness and toxicity. Int J Radiat Oncol Biol Phys 2011;81(03):e111-e118

7 Luo CB, Teng MM, Chang FC, Chang CY, Guo WY. Radiation carotid blowout syndrome in nasopharyngeal carcinoma: angiographic features and endovascular management. Otolaryngol Head Neck Surg 2008;138(01):86-91

8 Cohen J, Rad I. Contemporary management of carotid blowout. Curr Opin Otolaryngol Head Neck Surg 2004;12(02):110-115

9 Chaloupka JC, Putman CM, Citardi MJ, Ross DA, Sasaki CT. Endovascular therapy for the carotid blowout syndrome in head and neck surgical patients: diagnostic and managerial considerations. AJNR Am J Neuroradiol 1996;17(05):843-852

10 Lesley WS, Chaloupka JC, Weigele JB, Mangla S, Dogar MA. Preliminary experience with endovascular reconstruction for the management of carotid blowout syndrome. AJNR Am J Neuroradiol 2003;24(05):975-981

11 Warren FM, Cohen JI, Nesbit GM, Barnwell SL, Wax MK, Andersen PE. Management of carotid 'blowout' with endovascular stent grafts. Laryngoscope 2002;112(03):428-433

12 Simental A, Johnson JT, Horowitz M. Delayed complications of endovascular stenting for carotid blowout. Am J Otolaryngol 2003;24(06):417-419 